\title{
Influence of the ways of expression of the additivity of superficial protective film components on the estimation of their partial contributions to the total protective effect
}

\author{
R. V. Kashkovskiy \\ Gazprom VNIIGAZ LLC, Razvilka, Leninsky Rayon, Moscow Region, \\ 142717 Russian Federation \\ E-mail: roman.kashkovskiy@gmail.com
}

\begin{abstract}
As studies directed at improvement of a method for estimation of the amount to which corrosion products and inhibitor films contribute to the total protection effects are being held, various approaches to express the additivity of superficial protective film components are considered, along with their influence on the estimation of the partial contributions of each individual component of a superficial protective film. The latter will be discussed in our subsequent study.
\end{abstract}

Keywords: corrosion, additivity, contribution, inhibitor, steel, hydrogen sulfide, amines.

Received: March 13, 2014.

doi: $10.17675 / 2305-6894-2014-3-2-120-128$

\section{Introduction}

The key methodological issue in the estimation of partial contributions of a "corrosion products - inhibitor" superficial protective film, as it is pointed out in [1-3], is in their definition and taking into account their interactions. It is known that a film of corrosion products (CP) grows in an inhibited environment under the influence of an adsorbed inhibitor. At the same time, the emergence, growth, and structural changes which occur in a CP film with time [4] also influence the features of decelerator adsorption [5]. Taking into account the fact that a full account of this kind of interference is extremely difficult, a number of authors $[1,6]$ concluded that it was feasible to assume an additivity of the growing inhibitor and $\mathrm{CP}$ films (i.e., that there was no interaction).

Meanwhile, in a number of studies [1-3,7], the additivity of films was estimated on the basis of a series of assumptions on the additivity of protective effects $(Z)$ of system components only (in approach 1). However, this mathematical treatment of films additivity is highly disputable, thus the approach considering the additivity of protection coefficients $(\gamma)$ of superficial film components (in approach 2) appears to be more successful [8].

$$
Z_{\Sigma}=\sum_{i=1}^{n} Z_{i}
$$




$$
\gamma_{\Sigma}=\stackrel{n}{\mathrm{P}} \gamma_{i=1}
$$

where $Z$ and $\gamma$ indicate the inhibitor's protective effect and the corrosion protection coefficient, respectively; $P$ is the product, while $\Sigma$ and $i$ are interlinear indexes indicating the total and single indicators, respectively.

Expression (2) can be algebraically deduced on the basis of well-known theoretical regularities.

The corrosion protection coefficient in the inhibited environment $\left(\gamma_{i n}\right)$ is expressed as follows:

$$
\gamma_{\text {in }}=\frac{K_{0}}{K_{\text {in }}}
$$

where $K_{0}$ and $K_{\text {in }}$ indicate the corrosion rate in the background and inhibited environment, respectively.

The corrosion rate in the background environment is defined by presence of CP films on metal surfaces only. Corrosion rate decreases in time if the CP film possesses protective properties and vice versa, thus $K_{0}=K_{\mathrm{CP}}$. Arguing similarly, we obtain that $K_{\mathrm{in}}=K_{\Sigma}$ as both the $\mathrm{CP}$ and inhibitor films are present on metal in the inhibited environment $(\Sigma=\mathrm{CP}+$ in). Therefore,

$$
\frac{K_{0}}{K_{\text {in }}}=\frac{K_{\mathrm{CP}}}{K_{\Sigma}}
$$

Further, we express the $1 / K_{\Sigma}$ multiplier from formula (4) and then multiply both parts of the received equality by $K_{0}$ :

$$
\frac{K_{0}}{K_{\Sigma}}=\frac{K_{0}}{K_{\mathrm{CP}}} \cdot \frac{K_{0}}{K_{\text {in }}},
$$

According to (3), the latter expression represents a special case of equality (2), namely:

$$
\gamma_{\Sigma}=\gamma_{\mathrm{CP}} \cdot \gamma_{\text {in }} \cdot
$$

Then one attains the equality (7) expressing $\gamma_{\text {in }}$ from the formula (6):

$$
\gamma_{\text {in }}=\gamma_{\Sigma} / \gamma_{\mathrm{CP}}
$$

The attained formula (7) is transformed by substitution of the expression relating $\gamma$ and $Z$ :

$$
\gamma=\frac{1}{1-Z}
$$

so one attains in this case: 


$$
\frac{1}{1-Z_{\text {in }}}=\frac{1-Z_{\mathrm{CP}}}{1-Z_{\Sigma}} .
$$

After this, we find the $Z_{i n}$ value for $\gamma_{i}$ additivity case:

$$
Z_{\text {in }}=1-\frac{1-Z_{\Sigma}}{1-Z_{\mathrm{CP}}}=\frac{Z_{\Sigma}-Z_{\mathrm{CP}}}{1-Z_{\mathrm{CP}}} .
$$

From the attained expression (10) it follows that the distinction between the considered approaches consists of $1 /\left(1-Z_{\mathrm{CP}}\right)$ multiplier presence at the $Z_{\text {in }}$ calculation only. Therefore, $Z_{\mathrm{CP}}$ increases eventually on the condition of a protective film formation, while the CP film is formed, and, respectively, the $Z_{\text {in }}$ value increases as compared with the approach considering $Z_{i}$ additivity.

On the other hand, the $1 /\left(1-Z_{\mathrm{CP}}\right)$ factor tends to 1 if the $\mathrm{CP}$ film does not possess protective functions, leaving a factor which does not influence the change in the $Z_{\text {in }}$ value, which thus tends to $Z_{\Sigma}$. Both approaches produce similar estimation results only in this case.

Thus, the subject matter of this research was the definition of the influence of ways of expression of additivity of the interaction of superficial films towards the distribution their contributions to the total protective effect.

The above stated influence is studied using the example of the inhibition of hydrogen sulfide corrosion (HSC) of low carbon steel in the following work. Procedures of the tests carried out corresponded fully to earlier described techniques [3,9] and therefore do not need to be mentioned in the work further. The corrosion tests results used were attained and analyzed in the corresponding publications in detail $[6,10-11]$.

\section{Results and Discussion}

Comparison of the estimation results was carried out using initial [1] and modified methods $[2,3]$ considering reduction of the CP film thickness in the inhibitor environment and its dissolution with assumptions on the additivity of protective effects, $Z$, (in approach 1 ) and corrosion protection coefficients, $\gamma$, (in approach 2) of a superficial protective film components.

The results of estimations on surface component contributions for various methods of additivity conception were calculated using the initial method [1] and are presented in Fig. 1. As indicated earlier [1,7], $Z_{\mathrm{CP}}$ is, in this case, directly defined from the $K_{0}$ kinetics, and therefore does not depend on methods of additivity conception. Results attained in this work (curve 1 , Fig. $1 a$ and $1 b$ ) also confirm this. $Z_{\Sigma}$, in its turn, is defined from the $K_{\text {in }}$ kinetics and the $K$ value at an initial time point, i.e., it does not depend on methods of additivity expression, as well (curves 5-7, Fig. 1). 

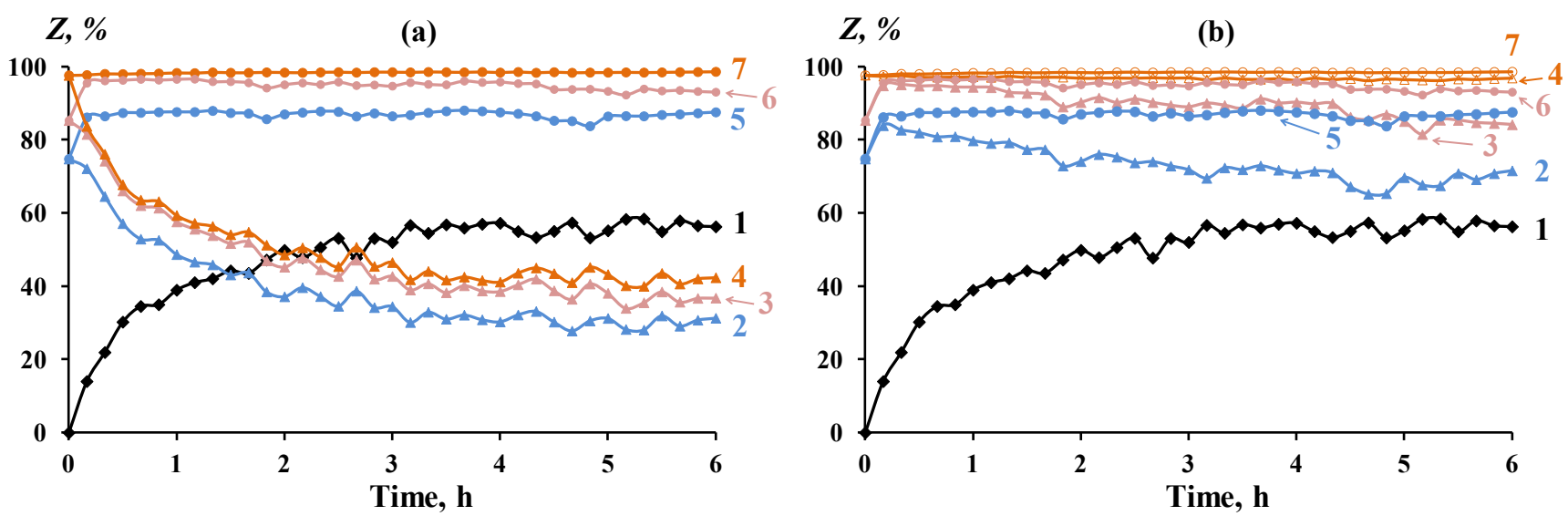

Figure 1. Corrosion products ( 1 ) and inhibitor films contributions (2-4) to total protective effect $(5-7)$, calculated by means of original method [1] with assumptions of the additivity of protective effects $(a)$ or corrosion protection coefficients $(b)$ of superficial protective film components. St3 steel grade, $\mathrm{H}_{2} \mathrm{~S}$ continuous flow. $C_{\text {in }}=50(2,5), 500(3,6)$, or $2000 \mathrm{mg} / \mathrm{L}(4,7)$.

According to expression (10), $Z_{\text {in }}$ has to be slightly higher in the case of additivity of $\gamma_{i}$ than that under the assumption of $Z_{i}$ additivity, considering that the polysulfide film is capable of slowing down HSC $\left(Z_{\mathrm{CP}}>0\right)[9,12]$. This is proved by the results of the performed estimation (curves $2-4$ in Fig. $1 a$ and $1 b$ ). As one would expect, the $Z_{\text {in }}$ value increases with an increase in $C_{\text {in }}$.

Thus, the contribution of the inhibitor film is the most dominant when estimating contributions according to the initial method with the consideration of $\gamma_{i}$ additivity, while $\mathrm{CP}$ film has the dominant contribution according to a consideration of $Z_{i}$ additivity.

However, as it is noted in [3], the initial method is not entirely correct in its estimation of the protective action of $\mathrm{CP}$ film in the inhibited environment, equalizing it with $Z_{\mathrm{CP}}$ in a background by their efficiency. Meanwhile, the formed CP film has a smaller thickness in the inhibited environment due to the delayed mechanism of inhibitor action, and the $Z_{C P}$ value in the inhibited environment has to be reasonably less than that in the background, allowing for an absence of superficial film interaction. This phenomenon can be considered in the contributions estimation using the chart showing the kinetics of specific losses of metal mass in background and inhibited environments and also the kinetics of the protective efficiency of sulfide film in background [3].

On the other hand, CP film can be dissolved in a liquid phase, and exfoliate from metal at the achievement of certain critical film thickness. It is necessary to carry out a quantitative analysis of the CP maintenance in a solution and contribution (in case of its existence) for the purpose of $Z_{\mathrm{CP}}$ estimation correction. In this case, data from specific losses of metal mass minus an amount of metal passed into electrolyte, due to film dissolution, are put on the above described intermediate chart. These calculations, also given in [2], are the basis of the modified method of estimation of contributions of superficial protective system components to $Z_{\Sigma}$. 
Results for the estimation of the $Z_{\mathrm{CP}}$ and $Z_{\text {in }}$ values at various $C_{\text {in }}$ using the modified method with consideration to different approaches to an interpretation of interaction additivity are given in Figs. 2-4.
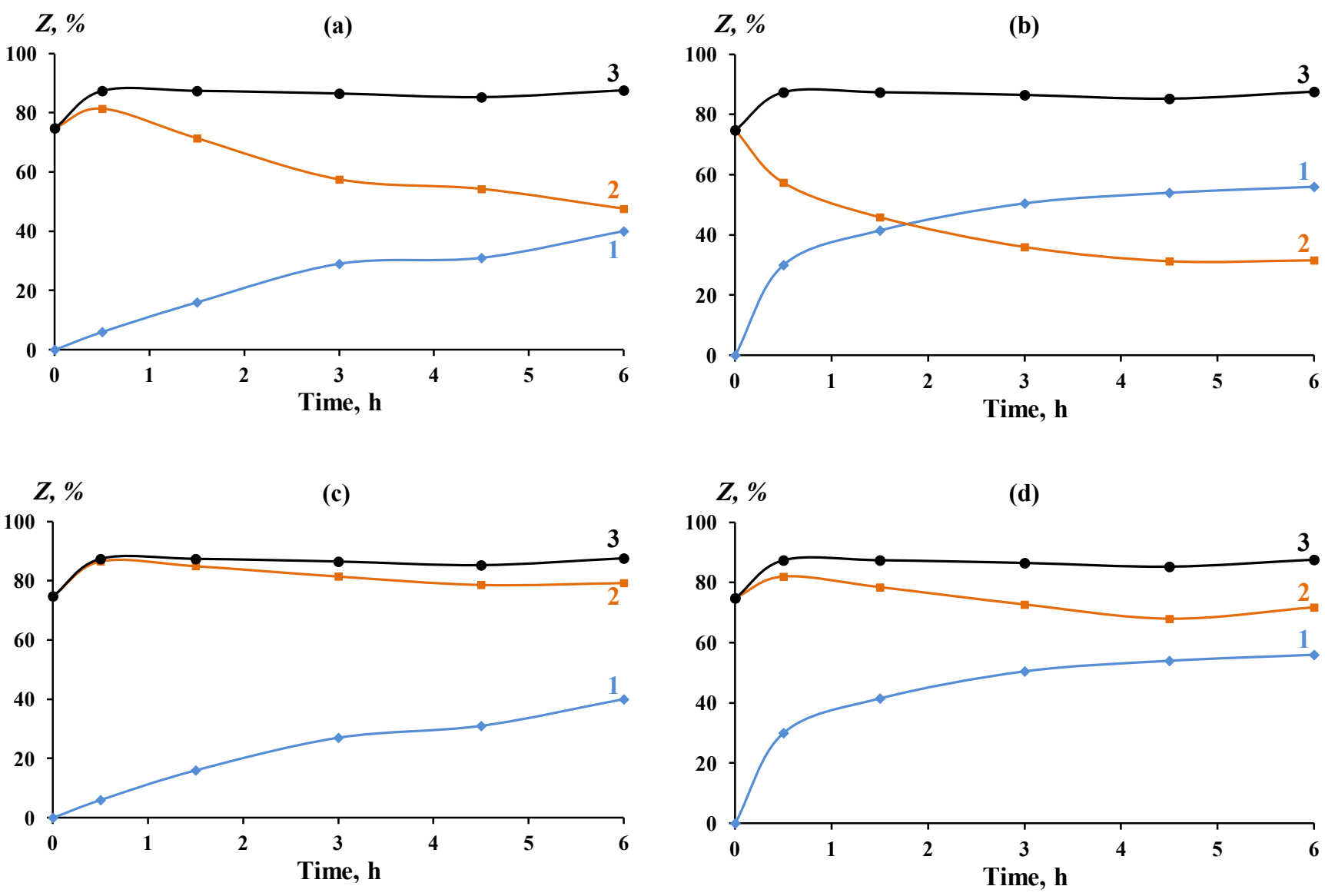

Figure 2. Corrosion products ( 1 ) and inhibitor films contributions (2) to the total protective effect (3), calculated by means of taking into consideration the reduction of $\mathrm{CP}$ film thickness $(a, c)$ and its dissolution $(b, d)$ with assumptions of the additivity of protective effects $(a, b)$ or corrosion protection coefficients $(c, d)$ of superficial protective film components. $C_{\mathrm{in}}=50 \mathrm{mg} / \mathrm{L}, \mathrm{St} 3$ steel grade, $\mathrm{H}_{2} \mathrm{~S}$ continuous flow.

It is expected, considering that the decrease in the thickness of a $\mathrm{CP}$ film and dissolution of this film have opposite impacts on the $Z_{\mathrm{CP}}$ value, that this will affect the results of contributions estimation. Thus, approach 1 leads to the opposite conclusions on the dominating contribution for $50 \mathrm{mg} / \mathrm{L}$ of inhibitor: $Z_{\text {in }}$ dominates in the case of taking into account the decrease in CP film thickness only, and, on the contrary, $Z_{\mathrm{CP}}$ prevails after 2 hours of exposure in case of an additional control of iron sulfide film dissolution (Fig. $2 a$ and $2 b)$.

Approach 2 qualitatively changes the situation of contributions distribution, but the tendencies for distribution change remain the same: $Z_{\mathrm{CP}}$ increases and $Z_{\text {in }}$ decreases with 
accounting for both specified factors, however, the contribution of $Z_{\text {in }}$ dominates in each case (Fig. $2 c$ and $2 d$ ).
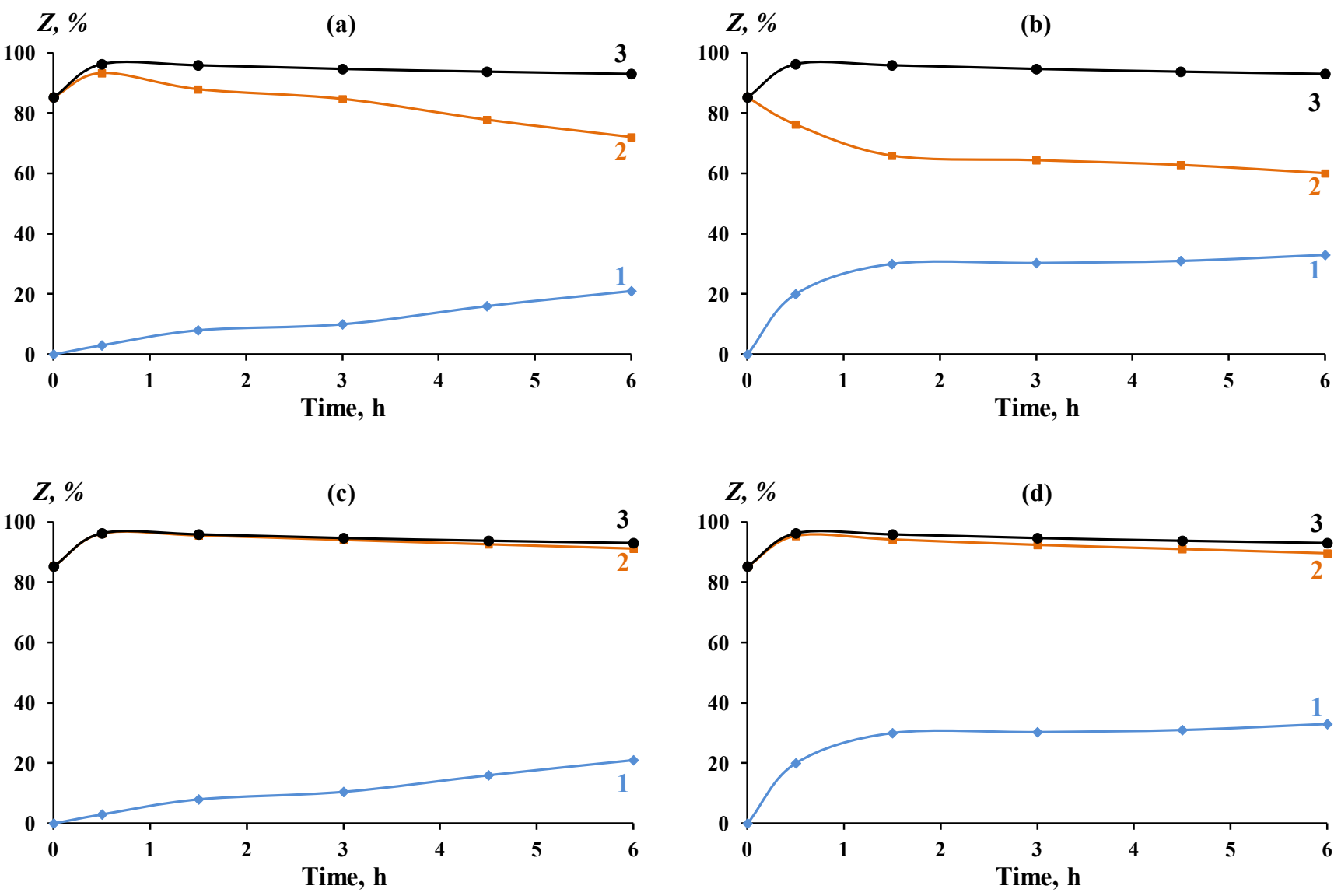

Figure 3. Corrosion products ( 1 ) and inhibitor films contributions (2) to the total protective effect (3), calculated by means of taking into consideration the reduction of CP film thickness $(a, c)$ and its dissolution $(b, d)$ with assumptions of the additivity of protective effects $(a, b)$ or corrosion protection coefficients $(c, d)$ of superficial protective film components. $C_{\text {in }}=500 \mathrm{mg} / \mathrm{L}, \mathrm{St} 3$ steel grade, $\mathrm{H}_{2} \mathrm{~S}$ continuous flow.

$Z_{\Sigma}$ and $Z_{\text {in }}$ naturally increase with $C_{\text {in }}$ growth to $500 \mathrm{mg} / \mathrm{L}$, and $Z_{\mathrm{CP}}$, on the contrary, decreases with respect to the values obtained at smaller $C_{\mathrm{in}}$, but the tendencies of contributions change remain the same (Fig. 3). It should also be noted that $Z_{\text {in }} \approx Z_{\Sigma}$ in this case, if the results of contributions estimation are based on method 2. Moreover, the $Z_{\text {in }}$ contribution continues to grow with a further growth in $C_{\mathrm{in} \text {, and almost completely reaches }}$ the $Z_{\Sigma}$ values expressed by means of protective effect value (curves 2 and 3 in Figs. $4 c$ and $4 d$ ). It is logical that when $Z_{\text {in }} \approx Z_{\Sigma}$, it would be more convenient to present the contributions on a $\lg \gamma=f(\tau)$ chart at such high concentrations of inhibitor $(2000 \mathrm{mg} / \mathrm{L})$ as it is shown in Fig. 5. 

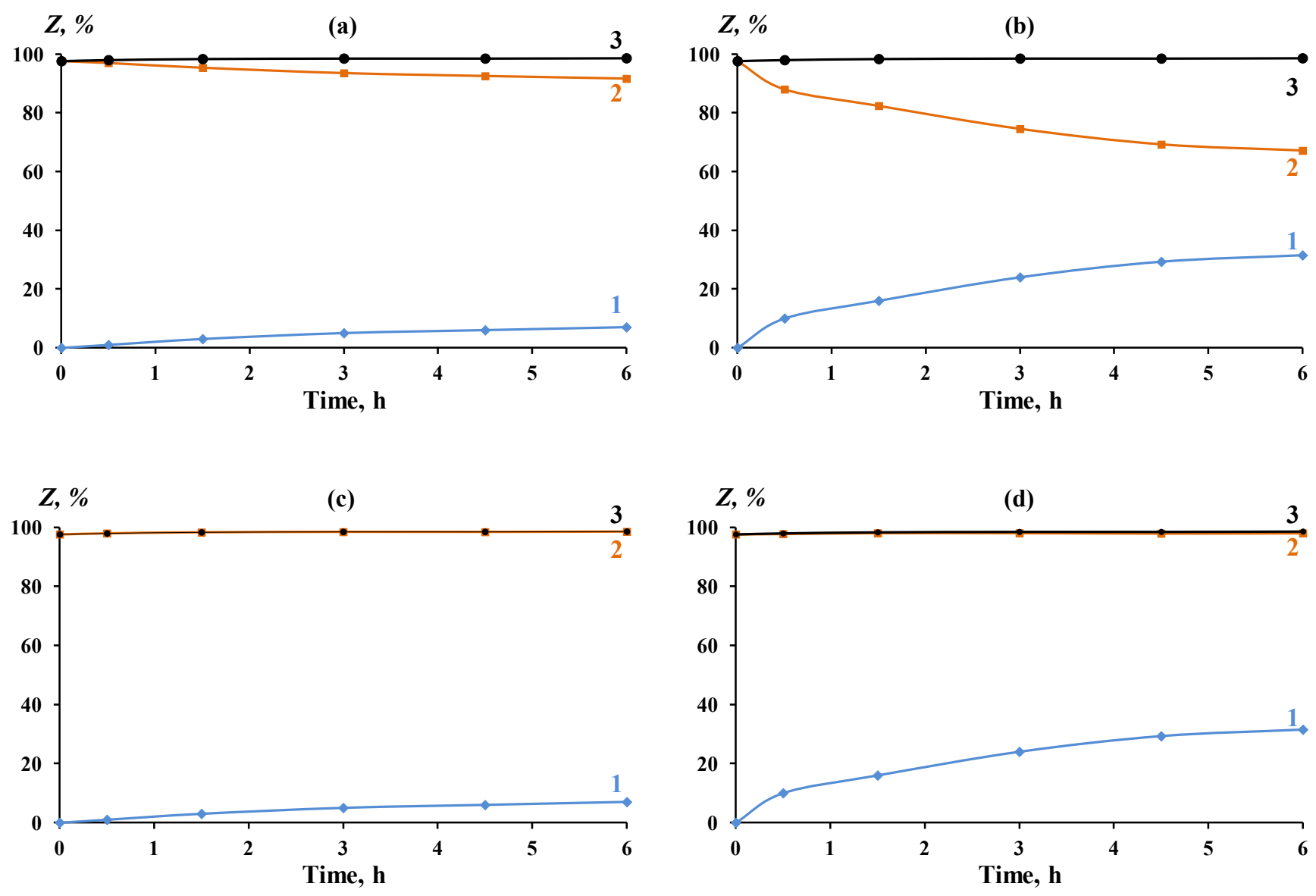

Figure 4. Corrosion products ( 1 ) and inhibitor film contributions (2) to the total protective effect (3), calculated by means of taking into consideration the reduction of $\mathrm{CP}$ film thickness $(a, c)$ and its dissolution $(b, d)$ with assumption of the additivity of protective effects $(a, b)$ or corrosion protection coefficients $(c, d)$ of superficial protective film components. $C_{\text {in }}=2000 \mathrm{mg} / \mathrm{L}, \mathrm{St} 3$ steel grade, $\mathrm{H}_{2} \mathrm{~S}$ continuous flow.

The $Z_{\mathrm{CP}}$ contribution, in its turn, significantly depends on the account of CP film thickness and its dissolution, and it weakly depends on the way of expression of component additivity. On the contrary, the $Z_{\text {in }}$ value strongly depends on the approach of additivity expression, and it more weakly depends on the account of $\mathrm{CP}$ film thickness changes. It should be noted that the approach considering the additivity of $\gamma_{i}$ of protective film components leads to an increase in the dominating contribution value, and this influence continues to amplify with a growth in contributions value itself. 

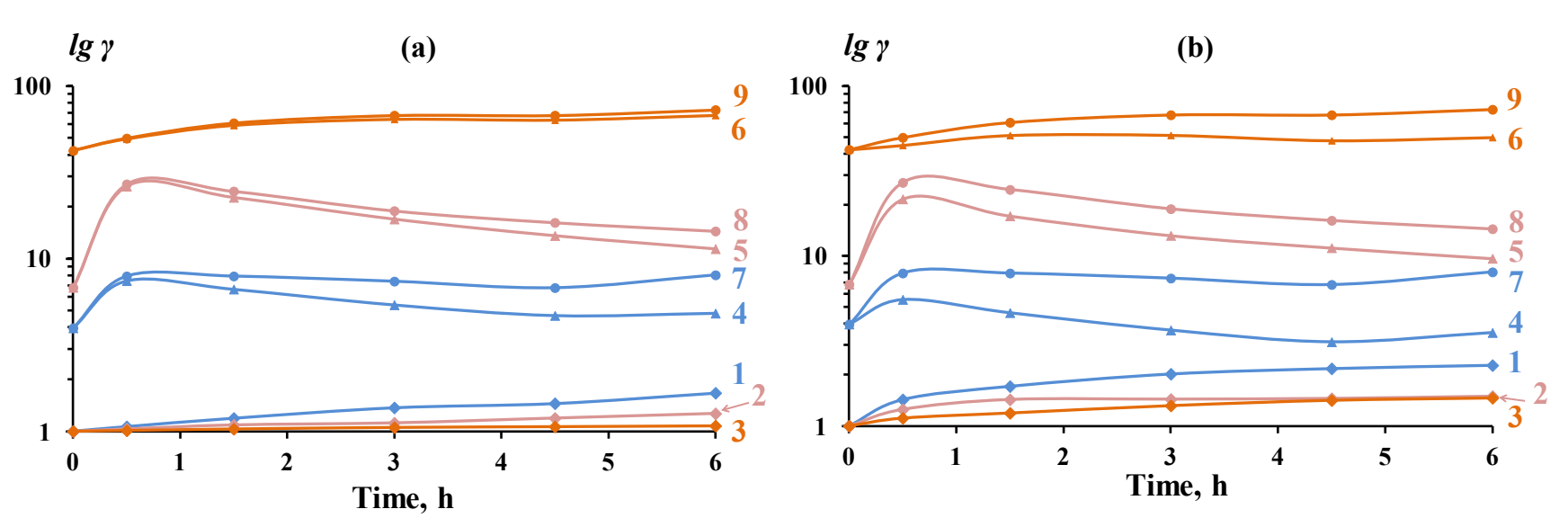

Figure 5. Corrosion products (1-3) and inhibitor films contributions (4-6) to the total protective effect (7-9), calculated by means of taking into consideration the reduction of CP film thickness $(a)$ and its dissolution $(b)$ with assumptions of the additivity of corrosion protection coefficients of superficial protective film components. $C_{\mathrm{in}}=50(1,4,7), 500(2,5$, $8)$ and $2000 \mathrm{mg} / \mathrm{L}(3,6,9)$, St3 steel grade, $\mathrm{H}_{2} \mathrm{~S}$ continuous flow.

\section{Conclusion}

1. A modified method of the estimation of superficial film component contributions to the total protective effect based on application of approach accounting additivity of films corrosion protection coefficients is offered. Comparison of the results of several estimation methods is carried out using the example of hydrogen sulfide corrosion inhibition of steel in a liquid phase.

2. It is shown that the account of additivity of corrosion protection coefficients of system components leads to a significant growth in the dominating contribution value, and the contribution of a corrosion inhibitor film is the dominating one in the case of hydrogen sulfide corrosion inhibition.

3. The $Z_{\Sigma}$ value does not depend on the account of the reduction of sulfide film thickness, its dissolution, and the way of expression of additivity of superficial protective film components.

4. The $Z_{\mathrm{CP}}$ contribution significantly depends on the account of $\mathrm{CP}$ film thickness, its dissolution, and it weakly depends on the way of expression of component additivity. The $Z_{\text {in }}$ value strongly depends on approach to additivity expression, and it more weakly depends on the account of CP film thickness changes.

\section{Acknowledgement}

The author wishes to express his sincere appreciation to Prof. Dr. Yu. Kuznetsov and Dr. N. Andreev (both from the Institute of Physical Chemistry and Electrochemistry of the Russian Academy of Sciences, Moscow, Russia) for valuable advice and remarks made during the preparation of this manuscript. 


\section{References}

1. V. I. Vigdorovich, L. E. Tsygankova and N. V. Shel, Surface and Interface Analysis, 2010, 42, 626.

2. R. V. Kashkovskiy, Int. J. Corros. Scale Inhib., 2013, 2, no. 4, 269. doi: 10.17675/2305-6894-2013-2-4-269-276

3. R. V. Kashkovskiy, Yu. I. Kuznetsov and L. P. Kazansky, Corros. Sci., 2012, 64, 126.

4. I. L. Rozenfeld, L. V. Frolova and V. M. Brusnikina, Soviet Scientific Reviews, Section B. Chemistry reviews, Amsterdam, OPA Ltd., 1987, 8, 115.

5. M. K. Panov, M. D. Getmanskiy, E. Kh. Enikeev and M. N. Fokin, Protection of metals, 1989, 25, no. 4, 555.

6. R. V. Kashkovskiy and Yu. I. Kuznetsov, Int. J. Corros. Scale Inhib., 2012, 1, no. 2, 117. doi: $\underline{10.17675 / 2305-6894-2012-1-2-117-129}$

7. R. V. Kashkovskiy, Yu. I. Kuznetsov and R. K. Vagapov, Korroz.: mater., zashch., 2010, no. 6, 18 (in Russian).

8. V. V. Ekilik and O. V. Chikov, Zashch. Met., 1991, Vol. 27, no. 1, 72 (in Russian).

9. R. V. Kashkovskiy, Yu. I. Kuznetsov and R. K. Vagapov, Korroz.: mater., zashch., 2011, no. 1, 28 (in Russian).

10. R. V. Kashkovskiy, Yu. I. Kuznetsov and N. P. Andreeva, Korroz.: mater., zashch., 2012, no. 7, 19 (in Russian).

11. R. V. Kashkovskiy, L. P. Kazansky and Yu. I. Kuznetsov, Korroz.: mater., zashch., 2012, no. 9, 20 (in Russian).

12. R. V. Kashkovskiy, Yu. I. Kuznetsov and R. K. Vagapov, European Corrosion Congress 2010 - EUROCORR 2010 Moscow, 2010, 3546. 